

\section{Immunoglobulins G could prevent adherence of Candida albicans to polystyrene and extracellular matrix components}

\author{
Marie-Helene Rodier, Christine Imbert, Catherine Kauffmann-Lacroix, \\ Gyslaine Daniault and Jean-Louis Jacquemin
}

Unité de recherche en biologie parasitaire et fongique, Laboratoire de parasitologie et mycologie médicales, CHU La Milètrie, 86021 Poitiers Cedex, France

\begin{abstract}
Immunocompromised patients are at high risk of developing Candida infections. Although cellmediated immunity is generally believed to play the main role in defence against fungi, antibodies could also be effective in immune defence by different mechanisms of action. The adherence capacity of four strains of Candida albicans to polystyrene and to some extracellular matrix components was investigated after incubation of the yeasts with non-specific and specific anti-C. albicans $\lg$ G. Experiments were carried out using a colorimetric method based upon the reduction of XTT tetrazolium (2,3-bis[2-methoxy-4-nitro-5-sulfophenyl]-2H-tetrazolium-5-carboxanilide) by mitochondrially active blastospores in the presence of menadione. Incubation of the yeasts with lgG, specific or not, caused a decrease in the capacity for adherence to the surfaces studied. There was no significant effect of the specificity of the tested antibodies on the reduction of adherence capacity. In conclusion, total lgG could play a role in blocking the binding of C. albicans to host and medical device surfaces. These results suggest that regular survey of levels of total lgG in patients suffering from severe hypogammaglobulinaemia could be of interest for the prevention of systemic candidiasis.
\end{abstract}

\section{INTRODUCTION}

Candida albicans, a saprophyte of the human digestive tract, is frequently responsible for systemic infections in immunocompromised patients, even if other species of Candida are reported with increased frequency (Vincent et al., 1998). According to a wide-ranging American study, the rate of invasive fungal infections among hospital patients approximately doubled between 1980 and 1990, and the incidence of nosocomial candidaemia alone increased fivefold (BeckSagué \& Jarvis, 1993).

C. albicans possesses virulence factors that are required for the establishment of candidiasis, involved in processes such as adhesion, phenotypic switching and morphogenesis (Calderone \& Fonzi, 2001). Adhesion of the organism to mucosal epithelium is a prerequisite for colonization and is therefore regarded as the initial step in the process leading to infection. Furthermore, adhesion to endothelium and extracellular matrix (ECM) components are required for dissemination of C. albicans (Klotz, 1992). A number of ECM proteins bind to the yeast, including fibronectin (Klotz et al., 1994), laminin (Sakata et al., 1999), vitronectin and type I and IV collagens (Klotz et al., 1993). Moreover, C. albicans is

Abbreviation: ECM, extracellular matrix. also able to adhere to the surface of intravascular catheters, usually colonized by intra- or extraluminal migration of Candida spp. from the skin surface (Flanagan \& Barnes, 1998). Candidaemia occurs commonly in the presence of a colonized intravascular catheter (Rex, 1996).

Nevertheless, the ability of this yeast to cause human infectious disease relates more to the immunological status of the host than to obvious virulence factors produced by the fungus. C. albicans is an opportunist yeast that becomes a pathogen in hosts whose local or systemic immune functions are impaired, for whatever reason (Matthews \& Burnie, 1996). Neutropenia represents a crucial risk factor, because neutrophils are indispensable for antifungal immunity (van Spriel et al., 2001). Derangements in antibody immunity often accompany defective cellular immunity. The role of antibody immunity in fungal infections remains a controversial subject. Many in vivo or in vitro studies have provided evidence for or against the importance of antibody immunity to C. albicans (Casadevall, 1995).

Some authors have reported that antibody immunity against C. albicans may participate in host defence by preventing attachment (Han \& Cutler, 1995). Most in vitro studies have 
concerned the inhibition of attachment by antibodies that recognize cell-wall proteins (Masuoka et al., 1999; San Millan et al., 1996).

We study here the adherence of $C$. albicans to polystyrene and to ECM components after incubation of the yeast with $\operatorname{IgG}$ directed against cytoplasmic antigens of $C$. albicans, compared with its adherence after contact of the yeast with nonspecific IgG.

\section{METHODS}

Polyclonal antiserum. C. albicans strain 2091, obtained from the Pasteur Institute (Paris, France), was grown for $48 \mathrm{~h}$ at $37^{\circ} \mathrm{C}$ on Sabouraud's dextrose agar slants (Sanofi Diagnostics Pasteur). The cells were harvested in Tris-buffered saline (TBS: $140 \mathrm{mM} \mathrm{NaCl}, 10 \mathrm{mM}$ Tris/ $\mathrm{HCl}, \mathrm{pH} 7 \cdot 2)$, washed three times at $4{ }^{\circ} \mathrm{C}$ by centrifugation $(600 \mathrm{~g}$, $10 \mathrm{~min}$ ) and suspended to a final concentration of $10^{9} \mathrm{cells} \mathrm{ml}^{-1}$ in the same buffer. Cells were disrupted for $10 \mathrm{~min}$ in an MSK cell homogenizer (B. Braun) with glass beads $(0.45-0.55 \mathrm{~mm})$ with cooling under $\mathrm{CO}_{2}$. Disrupted yeast cells were centrifuged at $100000 \mathrm{~g}$ for $30 \mathrm{~min}$ at $4{ }^{\circ} \mathrm{C}$. The supernatant fluid represented the cytoplasmic extract and was used for immunization of rabbits.

Antiserum directed against the C. albicans cytoplasmic extract was obtained after six subcutaneous injections, at 15 day intervals, of this extract at a concentration of $0.5 \mathrm{mg} \mathrm{ml}^{-1}$, emulsified (v/v) with QuilApurified saponin (Superfos Piosector), into three New Zealand white rabbits (Hy/Cr, Charles River Laboratories). After immunization, the three sera were pooled and the specificity of the resulting antiserum was verified by immunoblotting and indirect immunofluorescence assay.

IgG purification. Purification of the rabbit IgG before and after immunization was achieved with HiTrap Protein A columns (Pharmacia). The column was pre-equilibrated with $100 \mathrm{mM}$ Tris/ $\mathrm{HCl}, \mathrm{pH}$ 7.5. The pooled antiserum sample was diluted 1:1 with this buffer and filtered $(0.22 \mu \mathrm{m})$ prior to its application to the Protein A column, so that the optimal ionic strength and $\mathrm{pH}$ for binding were maintained. The diluted serum was then applied to the column and was allowed to flow completely into the gel. The bound IgG was eluted with $100 \mathrm{mM}$ glycine buffer, $\mathrm{pH} 2 \cdot 7$. Elution of bound proteins was monitored by measuring the $A_{280}$. Pooled IgG obtained from the rabbits before and after immunization was tested by an indirect immunofluorescence test with blastospores of C. albicans in order to confirm the effect of immunization and to determine the titre of the specific antibodies.

Organisms and growth conditions. C. albicans strains 1066, 2091 and NIH 311 (Pasteur Institute) and a clinical isolate (165-CA) obtained from blood-stream culture of a patient were used for this study. Identification of isolate 165-CA was performed by conventional physiological and morphological studies such as the germ-tube test in serum, agglutination (Bichro-Latex; Fumouze), metabolic properties (API 20C, API ID 32C; bioMérieux) and growth on Staib agar as described previously (Al Mosaid et al., 2001).

Yeasts were first grown for $48 \mathrm{~h}$ at $28^{\circ} \mathrm{C}$ on Sabouraud's agar slants (Sanofi Diagnostics Pasteur) to obtain a fresh culture of synchronous stationary-phase yeast. A loopful of this culture was transferred to $25 \mathrm{ml}$ yeast nitrogen base (Difco) supplemented with $300 \mathrm{mM}$ galactose (Sigma), which promotes adherence of C. albicans yeasts to cellular and plastic surfaces, and incubated without shaking for $36 \mathrm{~h}$ at $37^{\circ} \mathrm{C}$, as described previously (Imbert et al., 2002).

Incubation of blastospores with IgG. Prior to use for adherence experiments, blastospores were harvested and washed twice in $0 \cdot 1 \mathrm{M}$ PBS ( $\mathrm{pH} 7 \cdot 2$ ) (bioMérieux). Cell counts were determined and the suspension was adjusted to $10^{7}$ blastospores $\mathrm{ml}^{-1}$ and then incubated for $1 \mathrm{~h}$ at $37^{\circ} \mathrm{C}$ in PBS with the purified specific or non-specific IgG at different concentrations $\left(800,300,100\right.$ and $\left.50 \mu \mathrm{g} \mathrm{ml}^{-1}\right)$.

Protein concentration was determined as described by Bradford (1976) using BSA (Sigma) as a standard. A control consisted of incubating the yeasts in PBS alone.

Adherence of C. albicans to polystyrene. Adherence experiments were performed in untreated 96-well tissue-culture plates as described previously (Imbert et al., 2002). XTT tetrazolium (2,3-bis[2-methoxy4-nitro-5-sulfophenyl]-2H-tetrazolium-5-carboxanilide) was used to assess the adherence of C. albicans blastospores to wells of tissue-culture plates: the principle was based upon the reduction of XTT tetrazolium to XTT formazan by mitochondrially active C. albicans blastospores in the presence of an electron-coupling agent, menadione. Briefly, $C$. albicans blastospores were added, after pre-incubation with or without IgG at various concentrations, in culture plates as inocula of $10^{7}$ cells $\mathrm{ml}^{-1}$ in $150 \mu \mathrm{l}$ PBS alone for control or containing the IgG at the same concentrations. They were then allowed to adhere to polystyrene for $2 \mathrm{~h}$ at $37^{\circ} \mathrm{C}$; half of the wells were then washed twice with PBS to remove non-adherent yeasts. Thereafter, $300 \mu \mathrm{g}$ XTT ml ${ }^{-1}$ and $0.13 \mathrm{mM}$ menadione (both from Sigma) were added to all wells. Plates were incubated for $3 \mathrm{~h}$ at $37^{\circ} \mathrm{C}$ without shaking and then gently agitated and XTT formazan was measured by monitoring the $A_{492}$ in an LP400 micro-plate reader (Sanofi Diagnostics Pasteur) in washed and unwashed wells. The adherence capacity of each isolate was calculated as the mean absorbance in washed wells divided by the absorbance in unwashed wells.

Background formazan absorbance was determined with plates that contained PBS only or PBS, XTT and menadione; these values did not exceed 0.005 and were therefore not significant. All experiments were performed at least twice in six samples.

Adherence of C. albicans to ECM proteins. ECM gel and fibronectin (both from Sigma) were coated onto wells of 96-well tissue-culture plates (polystyrene; Evergreen Scientific) according to the manufacturer's instructions. ECM and fibronectin were used at a concentration of $10 \mu \mathrm{g} \mathrm{ml}^{-1}$. Aliquots of $250 \mu \mathrm{l}$ of each were coated onto wells overnight at $4{ }^{\circ} \mathrm{C}$. ECM gel was composed primarily of laminin, collagen type IV, heparan sulfate proteoglycan and entactin. Adherence of $C$. albicans to ECM proteins was determined in the same way as adherence to polystyrene, after the same steps of incubation, with or without the IgG at various concentrations.

Statistical analyses. Analysis of variance and Scheffe's test were conducted to determine differences among the test groups $(P<0 \cdot 05)$

\section{RESULTS}

\section{Verification of the immunization of rabbits}

Pooled sera from rabbits before and after immunization were tested by an immunofluorescence assay using C. albicans blastoconidia. The pooled serum obtained before immunization was negative, whereas the titre of serum obtained after immunization was $1: 1280$.

\section{Influence of IgG on adherence of $C$. albicans to different surfaces}

Incubation of blastospores with $\operatorname{IgG}$ directed against cytoplasmic extract of $C$. albicans at different concentrations 
Table 1. Effect of IgG on the capacity of four strains of C. albicans to adhere to polystyrene, fibronectin and ECM proteins

Adherence was determined as described in Methods. Non-specific (NS) and specific (S) IgG were used at the concentrations indicated. Control incubations contained PBS alone.

\begin{tabular}{|c|c|c|c|c|c|c|c|c|c|}
\hline \multirow[t]{2}{*}{ Strain } & \multirow[t]{2}{*}{ Control } & \multicolumn{2}{|c|}{$800 \mu \mathrm{g} \mathrm{ml}^{-1}$} & \multicolumn{2}{|c|}{$300 \mu \mathrm{g} \mathrm{ml}^{-1}$} & \multicolumn{2}{|c|}{$100 \mu \mathrm{g} \mathrm{ml}^{-1}$} & \multicolumn{2}{|c|}{$50 \mu \mathrm{g} \mathrm{ml}^{-1}$} \\
\hline & & NS & S & NS & S & NS & S & NS & S \\
\hline \multicolumn{10}{|c|}{ Polystyrene } \\
\hline 1066 & $48 \cdot 7 \pm 5 \cdot 1$ & $25 \cdot 2 \pm 4 \cdot 3^{a \star}$ & $24 \cdot 0 \pm 0 \cdot 7^{a}$ & $25 \cdot 3 \pm 0 \cdot 4^{a}$ & $24 \cdot 2 \pm 1 \cdot 1^{a}$ & $25 \cdot 2 \pm 0 \cdot 3^{a}$ & $25 \cdot 6 \pm 0 \cdot 3^{a}$ & $30 \cdot 4 \pm 3 \cdot 0^{a}$ & $28 \cdot 2 \pm 0 \cdot 2^{a}$ \\
\hline 2091 & $47 \cdot 2 \pm 1 \cdot 3$ & $17 \cdot 3 \pm 3 \cdot 0^{a}$ & $20 \cdot 3 \pm 3 \cdot 0^{a}$ & $18 \cdot 4 \pm 0 \cdot 9^{a}$ & $19 \cdot 6 \pm 1 \cdot 0^{a}$ & $16 \cdot 8 \pm 2 \cdot 1^{a}$ & $17 \cdot 4 \pm 0 \cdot 9^{a}$ & $30 \cdot 7 \pm 2 \cdot 3^{a}$ & $28 \cdot 2 \pm 3 \cdot 2^{a}$ \\
\hline NIH 311 & $71 \cdot 4 \pm 2 \cdot 5$ & $29 \cdot 3 \pm 6 \cdot 1^{a}$ & $27 \cdot 2 \pm 2 \cdot 5^{a}$ & $31 \cdot 6 \pm 0 \cdot 5^{a}$ & $28 \cdot 2 \pm 3 \cdot 2^{a}$ & $58 \cdot 2 \pm 7 \cdot 0^{a}$ & $58 \cdot 4 \pm 4 \cdot 2^{a}$ & $57 \cdot 5 \pm 0 \cdot 9^{a}$ & $61 \cdot 0 \pm 4 \cdot 3^{a}$ \\
\hline $165-\mathrm{CA}$ & $37 \cdot 7 \pm 1 \cdot 2$ & $30 \cdot 2 \pm 3 \cdot 6^{a}$ & $23 \cdot 3 \pm 2 \cdot 5^{a b}$ & $21 \cdot 2 \pm 1 \cdot 4^{a}$ & $25 \cdot 3 \pm 4 \cdot 0^{a}$ & $29 \cdot 4 \pm 3 \cdot 4^{a}$ & $32 \cdot 2 \pm 3 \cdot 4^{a}$ & $38 \cdot 1 \pm 3 \cdot 0^{a}$ & $35 \cdot 5 \pm 3 \cdot 7^{a}$ \\
\hline \multicolumn{10}{|c|}{ Fibronectin } \\
\hline 1066 & $67 \cdot 2 \pm 9 \cdot 2$ & $25 \cdot 7 \pm 4 \cdot 2^{a}$ & $23 \cdot 9 \pm 2 \cdot 1^{a}$ & $27 \cdot 2 \pm 3 \cdot 5^{a}$ & $23 \cdot 2 \pm 4 \cdot 3^{a}$ & $37 \cdot 2 \pm 3 \cdot 2^{a}$ & $36 \cdot 4 \pm 2 \cdot 7^{a}$ & $49 \cdot 7 \pm 2 \cdot 1^{a}$ & $45 \cdot 3 \pm 4 \cdot 3^{a}$ \\
\hline 2091 & $25 \cdot 3 \pm 2 \cdot 7$ & $16 \cdot 4 \pm 2 \cdot 9^{a}$ & $17 \cdot 3 \pm 2 \cdot 7^{a}$ & $18 \cdot 2 \pm 1 \cdot 2^{a}$ & $17 \cdot 7 \pm 2 \cdot 7^{a}$ & $20 \cdot 1 \pm 1 \cdot 2^{a}$ & $19 \cdot 2 \pm 1 \cdot 5^{a}$ & $22 \cdot 3 \pm 1 \cdot 3$ & $21 \cdot 5 \pm 2 \cdot 3$ \\
\hline NIH 311 & $51 \cdot 4 \pm 4 \cdot 2$ & $33 \cdot 9 \pm 3 \cdot 8^{a}$ & $25 \cdot 9 \pm 2 \cdot 9^{a b}$ & $37 \cdot 2 \pm 2 \cdot 5^{a}$ & $35 \cdot 3 \pm 1 \cdot 7^{a}$ & $42 \cdot 3 \pm 1 \cdot 8$ & $41 \cdot 8 \pm 1 \cdot 5^{a}$ & $51 \cdot 2 \pm 1 \cdot 7$ & $49 \cdot 3 \pm 2 \cdot 1$ \\
\hline 165-CA & $55 \cdot 7 \pm 7 \cdot 8$ & $22 \cdot 2 \pm 3 \cdot 5^{a}$ & $25 \cdot 3 \pm 2 \cdot 7^{a}$ & $27 \cdot 2 \pm 3 \cdot 5^{a}$ & $28 \cdot 3 \pm 2 \cdot 1^{a}$ & $34 \cdot 1 \pm 1 \cdot 7^{a}$ & $35 \cdot 3 \pm 2 \cdot 5^{a}$ & $52 \cdot 3 \pm 4 \cdot 2$ & $51 \cdot 1 \pm 3 \cdot 7$ \\
\hline \multicolumn{10}{|c|}{ ECM proteins } \\
\hline 1066 & $51 \cdot 2 \pm 5 \cdot 2$ & $23 \cdot 2 \pm 7 \cdot 3^{a}$ & $20 \cdot 5 \pm 4 \cdot 2^{a}$ & $20 \cdot 9 \pm 3 \cdot 2^{a}$ & $21 \cdot 5 \pm 2 \cdot 7^{a}$ & $25 \cdot 0 \pm 3 \cdot 1^{a}$ & $27 \cdot 0 \pm 4 \cdot 1^{a}$ & $38 \cdot 0 \pm 1 \cdot 2^{a}$ & $39 \cdot 0 \pm 0 \cdot 5^{a}$ \\
\hline 2091 & $25 \cdot 2 \pm 1 \cdot 3$ & $20 \cdot 0 \pm 2 \cdot 7^{a}$ & $18 \cdot 2 \pm 3 \cdot 2^{a}$ & $21 \cdot 5 \pm 0 \cdot 2^{a}$ & $21 \cdot 2 \pm 0 \cdot 3^{a}$ & $21 \cdot 1 \pm 0 \cdot 7^{a}$ & $20 \cdot 8 \pm 1 \cdot 0^{a}$ & $21 \cdot 9 \pm 1 \cdot 1^{a}$ & $20 \cdot 0 \pm 0 \cdot 4^{a}$ \\
\hline NIH 311 & $58 \cdot 4 \pm 3 \cdot 7$ & $32 \cdot 7 \pm 4 \cdot 9^{a}$ & $34 \cdot 3 \pm 7 \cdot 5^{a}$ & $31 \cdot 2 \pm 3 \cdot 2^{a}$ & $34 \cdot 4 \pm 0 \cdot 7^{a}$ & $37 \cdot 5 \pm 1 \cdot 1^{a}$ & $38 \cdot 2 \pm 3 \cdot 2^{a}$ & $57 \cdot 1 \pm 0 \cdot 8$ & $57 \cdot 0 \pm 0 \cdot 7$ \\
\hline $165-\mathrm{CA}$ & $21 \cdot 0 \pm 1 \cdot 8$ & $21 \cdot 7 \pm 3 \cdot 9$ & $22 \cdot 6 \pm 4 \cdot 8$ & $22 \cdot 1 \pm 1 \cdot 5$ & $20 \cdot 4 \pm 1 \cdot 8$ & $19 \cdot 5 \pm 1 \cdot 2$ & $20 \cdot 2 \pm 1 \cdot 3$ & $23 \cdot 2 \pm 1 \cdot 0$ & $21 \cdot 3 \pm 1 \cdot 5$ \\
\hline
\end{tabular}

*Significant differences $(P \leqslant 0 \cdot 001)$ are indicated in comparison with: $a$, control; $b$, non-specific IgG. 
induced a significant decrease in the adherence of the four tested strains to polystyrene, fibronectin and ECM proteins $(P<0.001)$ when compared with the adherence capacity of these strains incubated with PBS only. Only low concentrations of $\operatorname{IgG}$ were unable to decrease adherence, in the case of strains 2091 and 165-CA to fibronectin and of strain NIH 311 to fibronectin and ECM gel. Moreover, for strain 165-CA, there was no effect of the presence of IgG (specific or not) on adherence to ECM protein. We found the same results after incubation of the yeasts with non-specific IgG from rabbits before immunization, in comparison with the same controls (Table 1).

\section{Influence of specific IgG on the capacity of $C$. albicans to adhere to different surfaces}

Regardless of the surface studied and the conditions used, we found only two significant cases where the specificity of the IgG for C. albicans antigens influenced (at the highest concentration) the capacity of adherence of the yeast: adherence of strain 165-CA to polystyrene and of strain NIH 311 to fibronectin (Table 1). In all the other cases, there was no significant difference between the adherence capacities of the strains incubated with specific or non-specific IgG, regardless of concentration.

\section{DISCUSSION}

In high-risk patients suffering from disseminated candidiasis, predisposition to infection has many components, including both immunodeficiency (humoral and cellular), related to the pathology itself, and the results of immunosuppression, related to treatment (Tsiodras et al., 2000). Delayed immune reconstitution represents an increased risk of infectious complications (LaRocco \& Burgert, 1997). The incidence of invasive fungal infections is particularly high in bone-marrow transplant recipients. The conditional regimen used to prepare the host is a major determinant of host tissue injury and may lead to mucositis diarrhoea, facilitating transmucosal arrival of blood-stream infections (van Burik \& Weisdorf, 1999). Invasive monitoring with intravascular catheters predisposes to colonization and infection with Candida spp. (Flanagan \& Barnes, 1998). In intensive-care units, patients are also immunosuppressed following major surgery, trauma, burns or corticosteroid administration. In these patients, this is often associated with a decrease in intestinal mucosal barrier function (Flanagan \& Barnes, 1998).

Adhesion events to endothelium and ECM components are required for dissemination of $C$. albicans. This process could begin by yeasts gaining access to the blood stream through gastrointestinal perorption, by seeding from the biofilm of a medical device or by inoculation consecutive to a trauma (Glee et al., 2001). For these reasons, adherence to implanted devices and to ECM are of great importance for development of the disease. The adhesins of C. albicans are diverse, reflecting the ability of the organism to colonize and invade a variety of host cells and tissues (Bailey et al., 1995).
It has been reported in some in vitro studies that antibody immunity may contribute to host defence by direct candidacidal activity, by providing opsonins for more efficient phagocytosis, by binding to immunomodulating polysaccharides, by neutralizing extracellular proteases and by inhibiting the yeast-to-mycelium transition, but also by preventing attachment (Casadevall, 1995).

Administration of immune serum has been protective in some animal studies, but not in others. Polyclonal preparations are complex mixtures of antibodies, differing in isotype and specificity. This may explain why antibody protection has been observed only in some studies (Casadevall, 1995). Specific antibodies to mannoproteins and hsp90 have been shown to be protective against murine candidiasis (Matthews \& Burnie, 1996; Cassone et al., 1995). Specific antibody induction has been investigated as an immunotherapeutic preventative measure (Han \& Cutler, 1995). Recently, oral administration of bovine anti-Candida antibodies has been used for passive immunization in allogeneic bone-marrow transplant recipients (Tollemar et al., 1999).

The ability of a protein to block adherence may have potential in ameliorating or eliminating disease associated with this organism (Klotz \& Smith, 1995), even if blocking of invasion of tissue by C. albicans in order to reduce infection has had modest success in several animal models dealing with different forms of candidiasis (Pendrak \& Klotz, 1995).

In this study, we have investigated the effect of specific and non-specific $\operatorname{IgG}$ at different concentrations on the capacity of C. albicans to adhere to polystyrene and ECM components. IgG does adsorb spontaneously to polymer surfaces both in vitro and in vivo (Tang et al., 1993; Inoue et al., 1997). It has also been demonstrated that a biochemical interaction occurs between fibronectin and the Fc portion of IgG (Rostagno et al., 2002). Antibodies used in this study were directed against a cytoplasmic extract of C. albicans. Nevertheless, these specific IgG were able to recognize proteins localized in the fungus cell wall, as proved by the immunofluorescence assay. We have shown that the presence of $\operatorname{IgG}$, specific or not, at concentrations close to the in vivo situation, reduced the capacity of $C$. albicans to adhere to polystyrene and ECM components. More interestingly, this study highlights the fact that the hypogammaglobulinaemia found in immunocompromised patients (Hammarström et al., 1994, 2000) could play a role in the dissemination of Candida infections, not only because of the decrease in specific antibodies, but also because total IgG could present a barrier to the interaction between pathogens and host components or medical device surfaces. This could have clinical implications: survey of the total level of immunoglobulins and maintenance of a sufficient amount of these immune proteins until the recovery of the immune system could have potential in the prevention of systemic candidiasis. 


\section{REFERENCES}

Al Mosaid, A., Sullivan, D., Salkin, I. F., Shanley, D. \& Coleman, D. C. (2001). Differentiation of Candida dubliniensis from Candida albicans on staib agar and caffeic acid-ferric citrate agar. J Clin Microbiol 39, 323-327.

Bailey, A., Wadsworth, E. \& Calderone, R. (1995). Adherence of Candida albicans to human buccal epithelial cells: host-induced protein synthesis and signaling events. Infect Immun 63, 569-572.

Beck-Sagué, C. \& Jarvis, W. R. (1993). Secular trends in the epidemiology of nosocomial fungal infections in the United States, 1980-1990. National Nosocomial Infections Surveillance System. J Infect Dis 167, 1247-1251.

Bradford, M. M. (1976). A rapid and sensitive method for the quantitation of microgram quantities of protein utilizing the principle of protein-dye binding. Anal Biochem 72, 248-254.

Calderone, R. A. \& Fonzi, W. A. (2001). Virulence factors of Candida albicans. Trends Microbiol 9, 327-335.

Casadevall, A. (1995). Antibody immunity and invasive fungal infections. Infect Immun 63, 4211-4218.

Cassone, A., Boccanera, M., Adriani, D., Santoni, G. \& De Bernardis, F. (1995). Rats clearing a vaginal infection by Candida albicans acquire specific, antibody-mediated resistance to vaginal infection. Infect Immun 63, 2619-2624.

Flanagan, P. G. \& Barnes, R. A. (1998). Fungal infection in the intensive care unit. J Hosp Infect 38, 163-177.

Glee, P. M., Cutler, J. E., Benson, E. E., Bargatze, R. F. \& Hazen, K. C. (2001). Inhibition of hydrophobic protein-mediated Candida albicans attachment to endothelial cells during physiologic shear flow. Infect Immun 69, 2815-2820.

Hammarström, L., Gardulf, A., Hammarström, V., Janson, A., Lindberg, K. \& Smith, C. I. E. (1994). Systemic and topical immunoglobulin treatment in immunocompromised patients. Immunol Rev 139, 43-70.

Hammarström, V., Pauksen, K., Svensson, H., Lönnqvist, B., Simonsson, B., Ringden, O. \& Ljungman, P. (2000). Serum immunoglobulin levels in relation to levels of specific antibodies in allogeneic and autologous bone marrow transplant recipients. Transplantation 69, 1582-1586.

Han, Y. \& Cutler, J. E. (1995). Antibody response that protects against disseminated candidiasis. Infect Immun 63, 2714-2719.

Imbert, C., Rodier, M. H., Daniault, G. \& Jacquemin, J. L. (2002). Influence of sub-inhibitory concentrations of conventional antifungals on metabolism of Candida albicans and on its adherence to polystyrene and extracellular matrix proteins. Med Mycol 40, 123-129.

Inoue, H., Fujimoto, K., Uyama, Y. \& Ikada, Y. (1997). Ex vivo and in vivo evaluation of the blood compatibility of surface-modified polyurethane catheters. J Biomed Mater Res 35, 255-264.

Klotz, S. A. (1992). Fungal adherence to the vascular compartment: a critical step in the pathogenesis of disseminated candidiasis. Clin Infect Dis 14, 340-347.
Klotz, S. A. \& Smith, R. L. (1995). Gelatin fragments block adherence of Candida albicans to extracellular matrix proteins. Microbiology 141, 2681-2684.

Klotz, S. A., Rutten, M. J., Smith, R. L., Babcock, S. R. \& Cunningham, M. D. (1993). Adherence of Candida albicans to immobilized extracellular matrix proteins is mediated by calcium-dependent surface glycoproteins. Microb Pathog 14, 133-147.

Klotz, S. A., Hein, R. C., Smith, R. L. \& Rouse, J. B. (1994). The fibronectin adhesin of Candida albicans. Infect Immun 62, 4679-4681.

LaRocco, M. T. \& Burgert, S. J. (1997). Infection in the bone marrow transplant recipient and role of the microbiology laboratory in clinical transplantation. Clin Microbiol Rev 10, 277-297.

Masuoka, J., Wu, G., Glee, P. M. \& Hazen, K. C. (1999). Inhibition of Candida albicans attachment to extracellular matrix by antibodies which recognize hydrophobic cell wall proteins. FEMS Immunol Med Microbiol 24, 421-429.

Matthews, R. \& Burnie, J. (1996). Antibodies against Candida: potential therapeutics? Trends Microbiol 4, 354-358.

Pendrak, M. L. \& Klotz, S. A. (1995). Adherence of Candida albicans to host cells. FEMS Microbiol Lett 129, 103-113.

Rex, J. H. (1996). Catheters and candidemia. Clin Infect Dis 22, 467-470.

Rostagno, A. A., Gallo, G. \& Gold, L. I. (2002). Binding of polymeric IgG to fibronectin in extracellular matrices: an in vitro paradigm for immune-complex deposition. Mol Immunol 38, 1101-1111.

Sakata, N., Yamazaki, K. \& Kogure, T. (1999). Identification of a 21 kDa laminin-binding component of Candida albicans. Zentbl Bakteriol 289, 217-225.

San Millan, R., Ezkurra, P. A., Quindós, G., Robert, R., Senet, J. M. \& Pontón, J. (1996). Effect of monoclonal antibodies directed against Candida albicans cell wall antigens on the adhesion of the fungus to polystyrene. Microbiology 142, 2271-2277.

Tang, L., Lucas, A. H. \& Eaton, J. W. (1993). Inflammatory responses to implanted polymeric biomaterials: role of surface-adsorbed immunoglobulin G. J Lab Clin Med 122, 292-300.

Tollemar, J., Gross, N., Dolgiras, N., Jarstrand, C., Ringdén, O. \& Hammarström, L. (1999). Fungal prophylaxis by reduction of fungal colonization by oral administration of bovine anti-Candida antibodies in bone marrow transplant recipients. Bone Marrow Transplant 23, 283-290.

Tsiodras, S., Samonis, G., Keating, M. J. \& Kontoyiannis, D. P. (2000). Infection and immunity in chronic lymphocytic leukemia. Mayo Clin Proc 75, 1039-1054.

van Burik, J. A. \& Weisdorf, D. J. (1999). Infections in recipients of blood and marrow transplantation. Hematol Oncol Clin North Am 13, 10651089.

van Spriel, A. B., van den Herik-Oudijk, I. E. \& van de Winkel, J. G. J. (2001). Neutrophil Fc $\gamma$ RI as target for immunotherapy of invasive candidiasis. J Immunol 166, 7019-7022.

Vincent, J. L., Anaissie, E., Bruining, H. \& 12 other authors (1998). Epidemiology, diagnosis and treatment of systemic Candida infection in surgical patients under intensive care. Intensive Care Med 24, 206-216. 